\title{
Predictive PDF Control in Shaping of Molecular Weight Distribution Based on a New Modeling Algorithm
}

\author{
Jinfang Zhang ${ }^{\mathrm{a}}$, Hong Yue ${ }^{\mathrm{b}, *}$, Jinglin Zhou ${ }^{\mathrm{c}}$ \\ ${ }^{a}$ School of Control and Computer Engineering, North China Electric Power University, \\ Beijing, 102206, P.R. China \\ ${ }^{b}$ Department of Electronic and Electrical Engineering, University of Strathclyde, \\ Glasgow, G1 1XW, UK \\ ${ }^{c}$ College of Information Science and Technology, Beijing University of Chemical \\ Technology, Beijing, 100029, P.R. China
}

\begin{abstract}
The aims of this work are to develop an efficient modeling method for establishing dynamic output probability density function (PDF) models using measurement data and to investigate predictive control strategies for controlling the full shape of output PDF rather than the key moments. Using the rational square-root (RSR) B-spline approximation, a new modeling algorithm is proposed in which the actual weights are used instead of the pseudo weights in the weights dynamic model. This replacement can reduce computational load effectively in data-based modeling of a high-dimensional output PDF model. The use of the actual weights in modeling and control has been verified by stability analysis. A predictive PDF model is then constructed based on which predictive control algorithms are established with the purpose to drive the output PDF towards the desired target PDF over the control process. An analytical solution is obtained for the non-constrained predictive PDF control. For the constrained predictive control, the optimal solution is achieved via solving a constrained nonlinear optimization problem. The integrated method of data-based modeling and predictive PDF control is applied to closed-loop control of molecular weight distribution (MWD) in an exemplar styrene polymerization process, through which the modeling efficiency and the merits of predictive control over standard PDF control are
\end{abstract}

\footnotetext{
*Corresponding author. Tel. +44 1415482098

Email addresses: jfzhang@ncepu.edu.cn (Jinfang Zhang), hong.yue@strath.ac.uk (Hong Yue), jinglinzhou@mail.buct.edu.cn (Jinglin Zhou)
} 
demonstrated and discussed.

Keywords: Probability density function (PDF), B-spline approximation, parameter estimation, model predictive control (MPC), molecular weight distribution (MWD)

\section{Introduction}

Polymers are materials composed of macromolecules with different chain lengths, and the macromolecular architectures govern the properties of polymers such as thermal properties, stress-strain properties, impact resistance, strength, and hardness that affect end-use applications [1, 2]. The molecular weight distribution (MWD) is a major index featuring molecular and compositional characteristics of a polymer, therefore is one of the most important variables to be controlled in industrial polymerization processes. Compared with most control systems that aim to drive the output variables to follow desired set points in time domain, the shaping of MWD is much more complicated in modeling and controller design since the term to be controlled is a distribution function that needs to follow a target distribution. The lack of on-line measurement of MWD is another main problem in closed-loop control of polymerization reactions. Sensors like infrared and Raman scattering, mainly measure monomer conversion, not polymer molecular weight. Soft measurement of MWD through mathematical modeling is still the primary means to provide online information on MWD for control purposes. Mathematical models of MWD developed based on reaction mechanisms are normally nonlinear and of high dimensions. In the past decades, a number of methods have been developed for MWD control in polymerization processes $[3,4,5,6,7,8,9,10,11]$. An earlier review on modeling and optimization of particulate polymerization processes is given in [12].

Like MWD control in polymerization processes, many other industrial processes also have the problems that the product quality to be controlled is closely linked to output variables that need to follow certain distribution patterns, for example, particle size distribution (PSD) control in polymerization processes $[13,14,15,16]$, pulp fibre length distribution control in paper industries [17], particulate process control in powder industries [18, 19], crystal size distribution (CSD) control in crystallization processes [20, 21, 22], crystal size and shape distribution control of protein crystal aggregation in biopharmaceutical production [23], flame temperature distribution control 
in furnace systems $[24,25]$, power PDF control in nuclear research reactors [26], and bubble size distribution control in flotation processes [27], to name a few. To tackle the control problems for such systems, the idea of output stochastic distribution control (SDC) or output PDF control has been proposed, in which the full shape of the output distribution is directly controlled $[28,29,30,31,32]$. Unlike mean-and-variance control and other momentbased control, output PDF control can be applied to systems with output function of arbitrary distribution shape and subject to non-Gaussian noise terms. The purpose of output PDF control is to drive the output PDF towards the desired target PDF over the control process. It is fundamentally difficult to control the output distribution with a limited number of control inputs. In this work, we hope to make progress to tackle this challenging task from both modeling and control point of view.

Developing a general dynamic model to describe output PDF as a function of control input is the first step towards closed-loop control. Developing a reliable output PDF model from measurement data of control input and output distribution for any practical systems is a very challenging task. Bspline models are often used in output PDF approximation. The major advantage of a B-spline PDF model is the decoupling of time domain and PDF definition domain in formulation. Several B-spline based PDF models are developed, among them the simplest one is the linear B-spline PDF model, where the output PDF is approximated by B-spline basis functions linearly combined together, that is , $\gamma(y, u)=\sum_{i=1}^{n} \omega_{i}(u) B_{i}(y)$, where $\gamma(y, u)$ is the output PDF defined in a bounded region $[a, b] ; y \in[a, b]$ is an independent variable in the probability distribution space (note $y$ is not used to describe the system output as normally appeared in control literature). $u$ is the control input. $B_{i}(y)(i=1 \cdots n)$ are the B-spline basis functions defined in a specific range. $\omega_{i}(u)$ is the weight associated with $B_{i}(y) . n$ is the number of basis functions used, increasing which will improve approximation accuracy but cost more computational efforts. Considering the example of MWD modeling in a polymerization process, $y$ can be used to stand for the chain length, $u$ is the manipulated variable such as the ratio of monomer and catalyst flows, $\gamma(y, u)$ is the MWD to be controlled. Linear B-spline models have been used in our earlier studies of MWD modeling and closed-loop control system design $[8,33]$.

One numerical issue of a linear B-spline model is that the weights can sometimes be negative in calculation, which is not acceptable for a PDF. An alternative square-root model is developed to address this issue [34]. The 
integration constraint of $\int \gamma(y, u) d y=1$ must always apply for any PDFs in its definition domain. To cope with this integration constraint, a rational B-spline model is proposed [35]. Combining the square-root model and the rational B-spline model together, the so-called rational square-root (RSR) Bspline model is developed [36], which guarantees both the non-negativeness nature of a PDF and the integration constraint.

$$
\sqrt{\gamma(y, u)}=\frac{\sum_{i=1}^{n} \omega_{i}(u) B_{i}(y)}{\sqrt{\sum_{i, j=1}^{n} \omega_{i} \omega_{j} \int_{a}^{b} B_{i}(y) B_{j}(y) d y}}
$$

In this work, the RSR B-spline PDF model is further investigated so as to improve the computational efficiency in the data-based modeling procedure. It is one of our major aims to develop an efficient modeling algorithm for establishing dynamic output PDF models using data of control input and output PDF. This is significantly different from most existing works in output PDF control that were developed based on assumed models.

Model predictive control (MPC) is a well-established advanced control technique and has been widely accepted in industry since its early development in late seventies. It is a family of control methods that make explicit use of process models to predict system output at a future horizon. The sequence of future control signals are calculated by minimizing a performance index, at each time instance, and only the first control signal of the sequence is applied to the process. A receding strategy is applied to repeat the model prediction and optimal control design at all control intervals. Introductions on linear and nonlinear MPC can be found from a number of review articles such as [37, 38, 39]. Applying MPC to output PDF control systems, however, is rather difficult since it is the time-varying distribution to be controlled other than a variable. There are only a few works dealing with shaping of MWD through MPC strategy. In [40], an MPC method is proposed to control the MWD and PSD in free-radical emulsion copolymerization indirectly through controlling key parameters such as average radius, particle size polydispersity index (PSPI), number-average molecular weight, and monomer conversion. In [41], the low-order moments of MWD in styrene polymerization in a CSTR is controlled by MPC with constraints on control input. In [11], predictive control is employed to achieve desired trends in average composition and molecular weight drift in copolymerization. None of the above work directly controls a full MWD with MPC, only certain features relevant to MWD are controlled. In our earlier work, a non-constrained MPC was attempted for 
MWD control [42]. In this work, it is aimed to develop a predictive control scheme to control the full shape of MWD by considering control constraints.

The rest of this paper is organized as follows. In Section 2, a new modeling algorithm is proposed which employs the rational square-root (RSR) B-spline model for output PDF approximation. The rationale of using the actual weights instead of the pseudo weights is discussed with a strict stability analysis. A predictive PDF model is established in Section 3, based on which two predictive PDF control strategies are developed in Section 4. Simulation study of an exemplar MWD control system in styrene polymerization is carried out in Section 5 to evaluate the modeling efficiency and the control performances under different strategies. Conclusions are given in Section 6 .

\section{Data-based model development using RSR B-spline PDF ap- proximation}

\subsection{RSR B-spline model for output PDF approximation}

An output PDF control system is normally a nonlinear dynamic system, for which a nonlinear dynamic weights model is expected. However, due to the distribution characteristic in output, development of a nonlinear output PDF model using input data and output PDF data is extremely difficult. A reasonable assumption can be made that near the operating point, there is no essential difference between the linear weights dynamic models in [17] and the nonlinear weights models in [32] with Lipschitz assumptions. In this work, linear dynamics in weights are considered and the discrete-time RSR B-spline PDF model can be represented as follows:

$$
\begin{aligned}
& V(k+1)=A V(k)+B u(k) \\
& \sqrt{\gamma(y, k)}=\frac{C(y) V(k)}{\sqrt{V^{\mathrm{T}}(k) E V(k)}}
\end{aligned}
$$

where

$$
\begin{aligned}
C(y) & =\left[B_{1}(y), B_{2}(y), \cdots, B_{n}(y)\right] \\
E & =\int_{a}^{b} C^{\mathrm{T}}(y) C(y) d y
\end{aligned}
$$

$A$ and $B$ are matrices of proper dimensions, $k$ is the sampling time. The $n$ B-spline basis functions in $C(y)$ cannot all be zeros simultaneously, therefore 
matrix $E$ is invertible. Here $V(k)=\left[\omega_{1}(k), \omega_{2}(k), \cdots, \omega_{n}(k)\right]^{\mathrm{T}}$ is called the pseudo weights vector in the RSR B-spline model since it cannot be uniquely determined from $\gamma(y, k)$. The actual weights vector is defined as [36]

$$
V_{r}(k)=\frac{V(k)}{\sqrt{V^{\mathrm{T}}(k) E V(k)}} .
$$

It is apparent that $V_{r}(k)^{T} E V_{r}(k)=1$ for all $k$.

To establish a complete dynamic model as in (2)-(3) using the data of control input and output PDF, one needs to calculate $V(k)$ to obtain the PDF approximation weights at each time $k$, and estimate all parameters in $A$ and $B$ to establish the weights dynamics. It can be seen from (3) that the pseudo weights vector is difficult to be determined since the model regarding $V(k)$ is nonlinear and also $V(k)$ is not unique, however, the actual weights vector, $V_{r}(k)$, can be uniquely calculated from the PDF function $\gamma(y, k)$ as explained in the following.

Left multiplying $C^{\mathrm{T}}(y)$ to both sides of (3) and then taking integration for $y$ on both sides leads to

$$
\int_{a}^{b} C^{\mathrm{T}}(y) \sqrt{\left.\gamma_{(} y, k\right)} d y=\int_{a}^{b} C^{\mathrm{T}}(y) C(y) d y V_{r}(k)=E V_{r}(k)
$$

As discussed earlier $E$ is invertible, therefore the actual weights vector can be calculated from the output PDF by

$$
V_{r}(k)=E^{-1} \int_{a}^{b} C^{\mathrm{T}}(y) \sqrt{\gamma(y, k)} d y
$$

While it is impossible to uniquely determine the pseudo weights, $V(k)$, from an output PDF, a straightforward solution to calculate the B-spline actual weights, $V_{r}(k)$, is provided in (8). The rationale of using $V_{r}(k)$ in replacing $V(k)$ is further discussed in the next section.

\subsection{Feasibility analysis on the use of actual weights}

Denoting $e(k)=V(k)-V_{g}$, where $V_{g}$ is the vector of the actual weights corresponding to the desired PDF $\gamma_{g}(y)$, i.e. $V_{g}^{T} E V_{g}=1$, the following error dynamic system can be established for model (2)-(3):

$$
e(k+1)=A e(k)+B u(k)+A V_{g}-V_{g}
$$


The purpose of controller design is to choose a control sequence $u(k)$ such that the system's output PDF follows a pre-specified continuous PDF $\gamma_{g}(y)$ as close as possible. Here $\gamma_{g}(y)$ is also defined on $[a, b]$ and is independent of $u(k)$. This is equivalent to choosing an augmented controller

$$
\bar{u}(k)=\left(B^{\mathrm{T}} B\right)^{-1} B^{\mathrm{T}}(A-I) V_{g}+u(k),
$$

such that $\sqrt{\gamma(y, \bar{u}(k))}$ follows $\sqrt{\gamma_{g}(y)}$ as close as possible. As a result, $\bar{u}(k)$ can be designed in the form of output feedback control for the error dynamic system (9),

$$
\begin{aligned}
\bar{u}(k) & =K \epsilon(k) \\
\epsilon(k) & =\int_{a}^{b}\left(\sqrt{\gamma(y, \bar{u}(k))}-\sqrt{\gamma_{g}(y)}\right) d y \\
& =\Sigma_{0}\left(V_{r}(k)-V_{g}\right)
\end{aligned}
$$

where $\Sigma_{0}=\int_{a}^{b} C(y) d y$.

The following Lemma is presented for stability analysis of controller (11) in which the actual weights vector is used.

Lemma 1. For a function $f(\alpha, \beta)=\sqrt{\alpha^{\mathrm{T}} E \alpha}-\sqrt{\beta^{\mathrm{T}} E \beta}, E>0$, there is a $\rho$ with $\|\rho\| \leq \frac{\lambda_{\max }(E)}{\sqrt{\lambda_{\min }(E)}}$ such that

$$
\|f(\alpha, \beta)\| \leq \rho\|\| \alpha\|-\| \beta\|\|
$$

where $\|\cdot\|$ is Euclidean norm. $\lambda_{\max }(E)$ and $\lambda_{\min }(E)$ are the absolute maximum and minimum eigenvalue of $E$, respectively.

Proof.

$$
\begin{aligned}
\|f(\alpha, \beta)\| & =\left\|\frac{\alpha^{\mathrm{T}} E \alpha-\beta^{\mathrm{T}} E \beta}{\sqrt{\alpha^{\mathrm{T}} E \alpha}+\sqrt{\beta^{\mathrm{T}} E \beta}}\right\| \\
& \leq \frac{\|E\|\left\|(\alpha-\beta)^{\mathrm{T}}(\alpha+\beta)\right\|}{\left\|\sqrt{\alpha^{\mathrm{T}} E \alpha}+\sqrt{\beta^{\mathrm{T}} E \beta}\right\|} \\
& \leq \frac{\lambda_{\max }(E)\left\|(\alpha-\beta)^{\mathrm{T}}(\alpha+\beta)\right\|}{\sqrt{\lambda_{\min }(E)}\left\|\sqrt{\alpha^{\mathrm{T}} \alpha}+\sqrt{\beta^{\mathrm{T}} \beta}\right\|} \\
& =\rho\|\| \alpha\|-\| \beta\|\|
\end{aligned}
$$


The feedback controller (11) is formulated on the actual weights and has the following stability Theorem.

\section{Theorem 1. Selecting}

$$
K=L \sqrt{V_{g}^{\mathrm{T}} E V_{g}}
$$

if there is an $L$ so that $A+B L \Sigma_{0}^{\mathrm{T}}$ is Hurwitz, then the asymptotical stability of the error dynamic system (9) can be guaranteed by feedback control (11).

Proof. Substituting (12) into (11), we have

$$
\epsilon(k)=\frac{\Sigma_{0} e(k)}{\sqrt{V_{g}^{\mathrm{T}} E V_{g}}}+\frac{\Sigma_{0} V(k) \Lambda_{1}}{\sqrt{V_{g}^{\mathrm{T}} E V_{g}}},
$$

where $\Lambda_{1}=\sqrt{\frac{V_{g}^{\mathrm{T}} E V_{g}}{V^{\mathrm{T}}(k) E V(k)}}-1$, and the error dynamic can be written as

$$
\begin{aligned}
e(k+1) & =A e(k)+B L \sqrt{V_{g}^{\mathrm{T}} E V_{g}} \epsilon(k) \\
& =\left(A+B L \Sigma_{0}\right) e(k)+B L \Sigma_{0} V(k) \Lambda_{1} .
\end{aligned}
$$

Since $G=A+B L \Sigma_{0}^{\mathrm{T}}$ is Hurwitz, there exists a unique positive definite symmetrical matrix $P$ that satisfies the following Lyapunov equation

$$
G^{\mathrm{T}} P G-P=-I .
$$

Choose the following Lyapunov function

$$
\pi(e(k))=e^{\mathrm{T}}(k) P e(k),
$$

then

$$
\begin{aligned}
\Delta \pi= & \pi(e(k+1))-\pi(e(k)) \\
= & -\|e(k)\|^{2}+2 e^{\mathrm{T}}(k) G^{\mathrm{T}} P B L \Sigma_{0} V(k) \Lambda_{1} \\
& +\left(B L \Sigma_{0} V(k) \Lambda_{1}\right)^{\mathrm{T}} P\left(B L \Sigma_{0} V(k) \Lambda_{1}\right) \\
\leq & -\|e(k)\|^{2}+2 \frac{\left\|G^{\mathrm{T}} P B L \Sigma_{0}\right\| \lambda\|e(k)\|^{2}}{\sqrt{\lambda_{\min }(E)}} \\
& +\frac{\left\|P B L \Sigma_{0}\right\|^{2} \lambda^{2}\|e(k)\|^{2}}{\lambda_{\min }(E)} .
\end{aligned}
$$


Moreover, if

$$
\|L\| \leq \frac{\left(-\mu_{1}+\sqrt{\mu_{1}^{2}+4 \nu}\right) \sqrt{\lambda_{\min }(E)}}{2 \nu}
$$

where $\mu_{1}=\left\|G^{\mathrm{T}} P B \Sigma_{0}\right\| \rho$ and $\nu=\left\|P B \Sigma_{0}\right\|^{2} \rho^{2}$, then $\Delta \pi \leq 0$, which means this feedback control system is asymptotically stable.

From Theorem 1, it is known that if matrix $G$ is Hurwitz, there exists an output feedback controller that is based on the actual weights. Therefore, when $V(k)$ in the controller is replaced by the actual weights, this controller can be taken as a special output feedback controller. In other words, when an output PDF model satisfies the condition that $G$ being stable, at each control interval, the actual weights can be used to replace the pseudo weights. This supports our idea of using the actual weights to construct the RSR B-spline PDF model for controller design.

\subsection{Parameterization algorithm for data-based PDF modeling}

Using the actual weights, the following output PDF system is proposed.

$$
\begin{aligned}
V_{r}(k+1) & =\bar{A} V_{r}(k)+\bar{B} u(k) \\
\sqrt{\gamma(y, k)} & =C(y) V_{r}(k)
\end{aligned}
$$

$\bar{A}$ and $\bar{B}$ are matrices of the same dimensions as $A$ and $B$ in (2). For the same input, this model can be regarded as 'practically equivalent' to model (2)-(3), i.e., in the transient process, the output error between these two models are within an acceptable small range, and in the steady state, their output values are the same. The use of 'practically equivalent model' or 'characteristic model' in real engineering control systems was proposed and discussed in [43]. In the rest of the paper, the new RSR B-spline model in (15)-(16) will be used in parameter estimation and controller design.

Denote

$$
f(y, k)=\sqrt{\gamma(y, k)}
$$

as a function representing the output PDF at each time $k$. From (15)-(16), the function of output PDF can be written as

$$
\begin{aligned}
f(y, k) & =C(y) V_{r}(k) \\
& =C(y)\left(I-z^{-1} \bar{A}\right)^{-1} \bar{B} u(k-1) .
\end{aligned}
$$


Expanding (18) brings the parameterized model

$$
\begin{aligned}
f(y, k)= & a_{1} f(y, k-1)+\cdots+a_{n} f(y, k-n) \\
& +C(y) D_{0} u(k-1)+C(y) D_{1} u(k-2)+\cdots \\
& +C(y) D_{n-1} u(k-n)
\end{aligned}
$$

Note each $D_{i}(i=0, \cdots, n-1)$ is an $n$-dimensional vector which can be written as $D_{i}=\left[d_{i 1}, d_{i 2}, \ldots, d_{i n}\right]^{\mathrm{T}}$.

Let

$$
\begin{aligned}
\psi(y, k)= & {[f(y, k-1), \cdots, f(y, k-n),} \\
& u(k-1) B_{1}(y), \cdots, u(k-1) B_{n}(y), \cdots, \\
& \left.u(k-n) B_{1}(y), \cdots, u(k-n) B_{n}(y)\right]^{\mathrm{T}},
\end{aligned}
$$

and write the vector of parameters as

$$
\begin{gathered}
\theta=\left[a_{1}, \cdots, a_{n}, d_{01}, \cdots, d_{0 n}, d_{11}, \cdots,\right. \\
\left.d_{1 n}, \cdots, d_{(n-1) 1}, \cdots, d_{(n-1) n}\right]^{\mathrm{T}},
\end{gathered}
$$

equation (19) can be rewritten into a compact form as

$$
f(y, k)=\theta^{\mathrm{T}} \psi(y, k) .
$$

When $n$ basis functions are used in the PDF approximation, the total number of parameters to be estimated in $\theta$ is $n \times(n+1)$. This suggests that model (20) is normally of high dimension in an output PDF control system.

Given the B-spline basis functions, least-square algorithms can be applied to recover the parameters in $\theta$ when the data of control input and output $\mathrm{PDF}$ is collected. Due to the large number of parameters included in the modeling, the following double-loop recursive least-square (RLS) algorithm is used to estimate $\theta$ by screening the PDF definition domain of $[a, b]$ for $y$ (inner loop, indexed by $i$ ) and going through the time domain for $k$ (outer loop):

$$
\begin{aligned}
\hat{\theta}(i, k) & =\hat{\theta}(i-1, k)+\frac{P(i-1, k) \psi(y(i), k) \varepsilon(i, k)}{1+\psi^{\mathrm{T}}(y(i), k) P(i-1, k) \psi(y(i), k)} \\
\varepsilon(i, k) & =f(y(i), k)-\theta^{\mathrm{T}}(i-1, k) \psi(y(i), k) \\
P(i, k) & =\left(I-\frac{P(i-1, k) \psi(y(i), k) \psi^{\mathrm{T}}(y(i), k)}{1+\psi^{\mathrm{T}}(y(i), k) P(i-1, k) \psi(y(i), k)}\right) P(i-1, k)
\end{aligned}
$$


The initial value of the RLS algorithm is set to be $\hat{\theta}(0,0)=\theta_{0}, P(0,0)=$ $10^{3-6} I_{n(n+1)}, P(0, k)=P(M, k-1)$ and $\hat{\theta}(0, k)=\hat{\theta}(M, k-1)$ for $k \geq 1 . M$ is the total number of samplings in the region of $[a, b]$.

The procedures for establishing the RSR B-spline PDF model can be summarized as follows.

S0: Initialize relevant terms at $k=0$. Define data set $\{y(i)\}, i=1, \cdots, M$, in the definition interval $[a, b]$ for $y$. Set $k=1$ for the recursive operation.

S1: At sampling time $k$, collect the input data $u(k)$ and the output PDF data $\gamma(y(i), k)$.

S2: Calculate $f(y(i), k)$ following (17).

S3: Estimate $\theta$ using the RLS algorithm in (21)-(23) for $i=1 \cdots M$.

S4: Increase $k$ to $k+1$, repeat $\mathrm{S} 1-\mathrm{S} 3$ until the end of the calculation.

The parameterized input-output model (19) can be used for output PDF control in general cases. However, this model is inadequate for predictive PDF control since there is no prediction function provided. In the next section, a predictive PDF model will be constructed to facilitate the development of predictive PDF control strategies.

\section{Construction of predictive PDF model}

Equation (19) can be written as

$$
f(y, k)=\sum_{i=1}^{n} a_{i} f(y, k-i)+\sum_{j=0}^{n-1} C(y) D_{j} u(k-j-1) .
$$

Introducing the back-shift operator $z^{-1}$, denote

$$
\alpha\left(z^{-1}\right)=1-\sum_{i=1}^{n} a_{i} z^{-i}, \beta\left(z^{-1}, y\right)=C(y) \sum_{j=0}^{n-1} D_{j} z^{-j}
$$

equation (24) can be represented as

$$
\alpha\left(z^{-1}\right) f(y, k)=\beta\left(z^{-1}, y\right) u(k-1) .
$$

The following Diophantine equation is introduced to construct the predictive PDF model

$$
1=G_{q}\left(z^{-1}\right) \alpha\left(z^{-1}\right)+H_{q}\left(z^{-1}\right) z^{-q}
$$


where $q$ is the index for model prediction steps, and

$$
G_{q}\left(z^{-1}\right)=1+\sum_{i=1}^{q-1} g_{q, i} z^{-i}, H_{q}\left(z^{-1}\right)=\sum_{j=0}^{n-1} h_{q, j} z^{-j} .
$$

Multiplying $G_{q}\left(z^{-1}\right)$ to both sides of (26) and taking into account the Diophantine equation (27), we have

$$
f(y, k+q)=H_{q}\left(z^{-1}\right) f(y, k)+G_{q}\left(z^{-1}\right) \beta\left(z^{-1}, y\right) u(k+q-1) .
$$

Define

$$
S_{q}\left(z^{-1}, y\right)=G_{q}\left(z^{-1}\right) \beta\left(z^{-1}, y\right)=C(y) \sum_{i=0}^{n-1+q-1} s_{q, i} z^{-i}
$$

and write

$$
\overline{\mathbf{C}}(y)=\left[\begin{array}{cccc}
C(y) & 0 & \cdots & 0 \\
0 & C(y) & \cdots & 0 \\
\vdots & \vdots & \cdots & \vdots \\
0 & 0 & \cdots & C(y)
\end{array}\right]
$$

When taking $q=1,2, \cdots, p$ ( $p$ is the model predictive horizon), the multistep predictive PDF model in (29) can be written into the following matrix format

$$
\Pi(y, k, p)=\hat{H} f(y, k)+\Omega(y) U(k)+\Phi(y) \eta(k),
$$


where

$$
\begin{aligned}
& \Pi(y, k, p)=\left[\begin{array}{c}
f(y, k+1) \\
f(y, k+2) \\
\vdots \\
f(y, k+p)
\end{array}\right], \quad \hat{H}=\left[\begin{array}{c}
H_{1}\left(z^{-1}\right) \\
H_{2}\left(z^{-1}\right) \\
\vdots \\
H_{p}\left(z^{-1}\right)
\end{array}\right], \\
& \Omega(y)=\overline{\mathbf{C}}(y)\left[\begin{array}{cccc}
s_{1,0} & 0 & \cdots & 0 \\
s_{2,1} & s_{2,0} & \cdots & 0 \\
\vdots & \vdots & \cdots & \vdots \\
s_{p, p-1} & s_{p, p-2} & \cdots & s_{p, p-l}
\end{array}\right] \\
& \Phi(y)=\overline{\mathbf{C}}(y)\left[\begin{array}{cccc}
s_{1,1} & s_{1,2} & \cdots & s_{1, n-1} \\
s_{2,2} & s_{2,3} & \cdots & s_{2, n-1+1} \\
\vdots & \vdots & \cdots & \vdots \\
s_{p, p} & s_{p, p+1} & \cdots & s_{p, n-1+p-1}
\end{array}\right] \text {, } \\
& U(k)=\left[\begin{array}{c}
u(k) \\
u(k+1) \\
\vdots \\
u(k+l-1)
\end{array}\right], \quad \eta(k)=\left[\begin{array}{c}
u(k-1) \\
u(k-2) \\
\vdots \\
u(k-n+1)
\end{array}\right] \text {. }
\end{aligned}
$$

$l(l \leq p)$ is the predictive control horizon. Equation (31) gives a compact format of the predictive output PDF model.

The coefficients in the Diophantine equation can be obtained by recursive development [44]. Here only the results are provided (detailed derivation can be found in many literature of standard MPC algorithms). With the initial setting of

$$
1=G_{1}\left(z^{-1}\right) \alpha\left(z^{-1}\right)+H_{1}\left(z^{-1}\right) z^{-1}
$$

where $G_{1}\left(z^{-1}\right)=g_{1,0}=1$ and $H_{1}\left(z^{-1}\right)=z\left(1-\alpha\left(z^{-1}\right)\right)$, the coefficients in $G_{q}\left(z^{-1}\right)$ is calculated by $g_{q+1, i}=g_{q, i}$. The coefficients in $H_{q}\left(z^{-1}\right)$ are 
calculated as follows for $q=1, \cdots, p$.

$$
\begin{aligned}
h_{q, 0} & =g_{q+1, q} \\
h_{q+1,0} & =h_{q, 1}+g_{q+1, q} \alpha_{1}=h_{q, 1}+h_{q, 0} \alpha_{1} \\
h_{q+1,1} & =h_{q, 2}+g_{q+1, q} \alpha_{2}=h_{q, 2}+h_{q, 0} \alpha_{2} \\
\vdots & \\
h_{q+1, n-2} & =h_{q, n-1}+g_{q+1, q} \alpha_{n-1}=h_{q, n-1}+h_{q, 0} \alpha_{n-1} \\
h_{q+1, n-1} & =g_{q+1, q} \alpha_{n}=h_{q, 0} \alpha_{n}
\end{aligned}
$$

Similarly, the coefficients in $S_{q}\left(z^{-1}, y\right)$ can be calculated recursively as follows.

$$
\begin{aligned}
s_{q+1, i} & =s_{q, i}+h_{q, 0} D_{i-q} \\
s_{q+1, n-1+q} & =h_{q, 0} D_{n-1}
\end{aligned}
$$

where $i=0,1, \ldots, n+q-2$, and $D_{m}=0$ when $m<0$. The initial settings are $s_{1, i}=D_{i}$ for $i=0, \cdots, n-1$.

Guidance on the selection of model predictive horizon, $p$, and control predictive horizon, $l$, can be found in many MPC literature. In principle, the selection of $p$ and $l$ needs to balance several performance requirements such as computational load, dynamic tracking performance, stability and robustness.

\section{Predictive PDF controller design}

In this section, predictive PDF control algorithms with and without control constraints are developed, respectively, based on the predictive PDF model (31). These MPC algorithms will be compared with a standard PDF controller, the latter is also briefed in the following.

\subsection{Standard output PDF control}

A general PDF control target is to drive the output PDF to follow the desired distribution. Using the following performance function

$$
J_{0}(u(k))=\int_{a}^{b}\left(\sqrt{\gamma(y, k)}-\sqrt{\gamma_{g}(y)}\right)^{2} d y+Q_{0} u^{2}(k)
$$


where $\gamma_{g}(y)$ is the target distribution, $Q_{0}$ is a weighting factor for control input, the optimal control input $u$ at time $k$ is obtained by taking $\frac{d J}{d u}=0$ to give $[17]$

$$
u(k)=\frac{\int_{a}^{b} C(y) D_{0} \bar{g}(y, k) d y}{\int_{a}^{b}\left(C(y) D_{0}\right)^{2} d y+Q_{0}}
$$

where

$$
\begin{aligned}
\bar{g}(y, k)= & -\sum_{i=2}^{n} a_{i} f(y, k-i+1)-C(y) D_{i-1} u(k-i+1) \\
& +\sqrt{\gamma_{g}(y)}-a_{1} f(y, k) .
\end{aligned}
$$

When the input-output model is established, at each time $k$, the controller design procedures can be summarized as follows.

S1: Formulate $\bar{g}(y, k)$ as in (37) using the parameterized PDF model (19).

S2: Calculate $\int_{a}^{b} C(y) D_{0} \bar{g}(y, k) d y$ and $\int_{a}^{b}\left(C(y) D_{0}\right)^{2} d y$.

S3: Obtain the optimal control input $u(k)$ with (36).

\subsection{Non-constrained predictive PDF control}

The following performance index is formulated in the first predictive PDF control algorithm

$$
\begin{aligned}
J(U(k))= & \int_{a}^{b}[\Pi(y, k, p)-\Gamma(y)]^{\mathrm{T}}[\Pi(y, k, p)-\Gamma(y)] d y \\
& +U^{\mathrm{T}}(k) Q U(k)
\end{aligned}
$$

where $\Gamma=\left[\sqrt{\gamma_{g}(y)}, \cdots, \sqrt{\gamma_{g}(y)}\right]^{\mathrm{T}}$ is the target PDF vector, $Q$ is the weighting matrix for control input.

Taking (31) into (38) and denoting

$$
\xi(y, k)=\hat{H} f(y, k)+\Phi(y) \eta(k)
$$

as the known term at time $k$, the optimization solution to (38) is derived from $\frac{d J}{d U}=0$ as follows:

$$
U(k)=-\left(\int_{a}^{b} \Omega^{\mathrm{T}}(y) \Omega(y) d y+Q\right)^{-1} \cdot\left(\int_{a}^{b} \Omega^{\mathrm{T}}(y)(\xi(y, k)-\Gamma(y)) d y\right) .
$$

Equation (40) gives an analytical solution for the $l$-step predictive controller when no constraints are considered in the optimization. 


\subsection{Constrained predictive PDF control}

Various constraints exist in real control systems such as upper and lower bounds of control input, varying rate in control input and output constraints. Failure in handling control constraints may lead to actuator saturation that will delay required control actions and decrease control performance. Constrained MPC is therefore widely needed in process control to prevent the manipulated variables from getting saturated or output going out of specific bounds.

Bounded constraints on control input are considered in this section to address actuator saturation or other operational constraints on manipulated variables. To simplify the discussion, constant upper and lower bounds are

set for control input as $u_{\min } \leq u(k) \leq u_{\max }$. Using the same predictive model as in (31) and the same quadratic performance function as in (38), the constrained MPC for output PDF control can be written as the following optimization problem:

$$
\begin{aligned}
J(U(k))= & \int_{a}^{b}[\Pi(y, k, p)-\Gamma(y)]^{\mathrm{T}}[\Pi(y, k, p)-\Gamma(y)] d y \\
& +U^{\mathrm{T}}(k) Q U(k) \\
\text { s.t. } & U_{\min } \preceq U(k) \preceq U_{\max }
\end{aligned}
$$

where $U_{\max }=\left[u_{\max }, \cdots, u_{\max }\right]^{T} \in \mathbb{R}^{l \times 1}$ and $U_{\min }=\left[u_{\min }, \cdots, u_{\min }\right]^{T} \in \mathbb{R}^{l \times 1}$ are vectors of upper and lower bounds, respectively.

The above predictive controller design with constraints is a nonlinear programming optimization problem, which do not have an analytical solution in general. Optimization methods like quadratic programming (QP), sequential quadratic programming (SQP), sequential unconstrained minimization technique, etc., can be applied to get the optimal solution. It can be seen from (41) and (38) that the two predictive control algorithms have the same optimization performance index, but the way to get the optimal solution is different since the constraints on control input are handled differently in these two algorithms.

\section{Simulation study of MWD shaping using predictive PDF control}

In this section, simulation studies on MWD shaping of a lab-scaled styrene polymerization process is carried out using the new RSR B-spline modeling and the two predictive PDF control algorithms. 


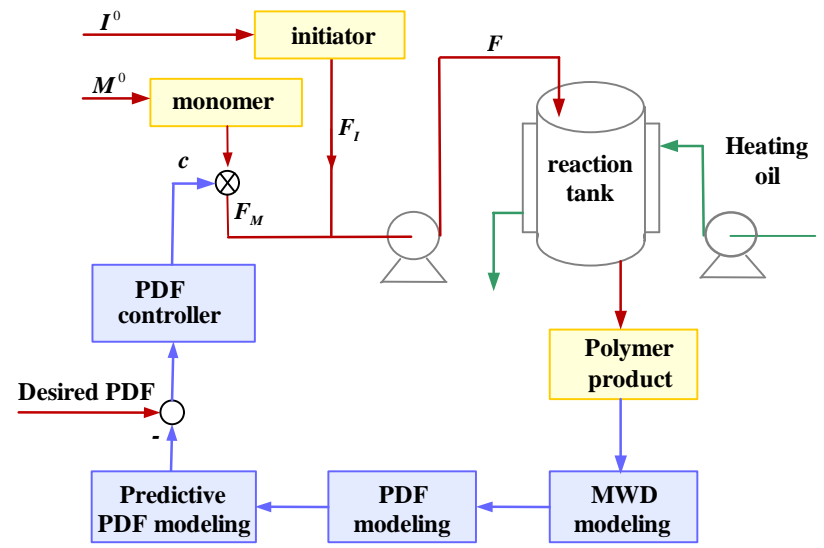

Figure 1: The sketch of the example polymerization system with predictive PDF control

\subsection{Dynamic model of $M W D$}

Consider a styrene polymerization process taking place in a semi-batch continuous stirring tank reactor (CSTR) (Fig. 1). The input flow $F$ to the tank is the sum flow rate of the monomer $\left(F_{M}\right)$ and the initiator $\left(F_{I}\right)$. The monomer and the initiator are fed into the reactor with a ratio of $C=$ $F_{M} /\left(F_{I}+F_{M}\right)$, which is used as the control input. The output is the MWD of the produced polymer. In the simulation, the sum flow rate $F$ is kept constant, only the ratio $C$ between the monomer and the initiator is adjusted.

The free radical polymerization mechanisms are considered which include initiation, chain propagation, chain transfer to monomer, and termination by combination. Under the framework of population balance, the generation function technique is used to derive the leading moments for MWD model, which are later developed into a parameterized model in the form of a SchultzZimm distribution. Full modeling details can be found from the authors' previous work [8]. The major ordinary differential equations (ODEs) are provided in the following to support the dynamic MWD description.

The concentration equations for initiator, $I$, and monomer, $M$, are described as

$$
\begin{aligned}
\frac{d I}{d t} & =\frac{F}{V}\left(I^{0}-I\right)-K_{d} I \\
\frac{d M}{d t} & =\frac{F}{V}\left(M^{0}-M\right)-2 K_{i} I-\left(K_{p}+K_{t r m}\right) M \psi_{0}
\end{aligned}
$$

where $I^{0}$ and $M^{0}$ are initial concentrations of initiator and monomer, respec- 
tively, in the input flow. $K_{d}$ is the initiator decomposition rate constant, $K_{i}$ is the initiation rate constant, $K_{p}$ is the propagation rate constant, and $K_{\text {trm }}$ is the chain transfer rate constant. $\psi_{0}$ is the total concentration of radicals.

Denote $\psi_{k}=\sum_{j=1}^{+\infty} j^{k} R_{j}(k=0,1,2)$ as the leading moments for radicals and $Z_{k}=\sum_{j=2}^{+\infty} j^{k} P_{j}(k=0,1,2)$ as the leading moments for dead polymer, where $R_{j}$ is the live polymer radical with chain length $j$ and $P_{j}$ is the dead polymer with chain length $j$. The following ODEs are derived using the generation function technique to give

$$
\begin{aligned}
\frac{d \psi_{0}}{d t} & =-\frac{F}{V} \psi_{0}+2 K_{i} I-K_{t} \psi_{0}^{2} \\
\frac{d \psi_{1}}{d t} & =-\frac{F}{V} \psi_{1}+2 K_{i} I+K_{p} \psi_{0} M-K_{t} \psi_{0} \psi_{1}+K_{t r m} M\left(\psi_{0}-\psi_{1}\right) \\
\frac{d \psi_{2}}{d t} & =-\frac{F}{V} \psi_{2}+2 K_{i} I+K_{p} M\left(2 \psi_{1}+\psi_{0}\right)-K_{t} \psi_{0} \psi_{2}+K_{t r m} M\left(\psi_{0}-\psi_{2}\right)
\end{aligned}
$$

where $K_{t}$ is the termination rate constant. The ODEs for the leading moments of polymers, $Z_{0}, Z_{1}, Z_{2}$, are derived to be

$$
\begin{aligned}
\frac{d Z_{0}}{d t} & =-\frac{F}{V} Z_{0}+K_{t r m} M_{o} \psi_{0}+\frac{K_{t}}{2} \psi_{0}^{2} \\
\frac{d Z_{1}}{d t} & =-\frac{F}{V} Z_{1}+K_{t r m} M_{o} \psi_{1}+K_{t} \psi_{0} \psi_{1} \\
\frac{d Z_{2}}{d t} & =-\frac{F}{V} Z_{2}+K_{t r m} M_{o} \psi_{2}+K_{t} \psi_{0} \psi_{2}+K_{t} \psi_{1}^{2}
\end{aligned}
$$

Instead of using higher-order moments to describe MWD of styrene polymerization product, a Schultz-Zimm distribution is regarded as an appropriate distribution function that can be established from those leading moments of polymer. A normalized Schultz-Zimme distribution function is defined by $[45]$

$$
f(n)=\frac{h^{h} n^{h-1} \exp \left(-h n / M_{n}\right)}{M_{n}^{h} \Gamma(h)},
$$

where $n \geq 0$ is the chain length, $h$ is a parameter indicating the distribution breadth, $M_{n}$ is the number average chain length which is defined as $M_{n}=Z_{1} / Z_{0}, \Gamma$ is the gamma function defined as $\Gamma(h)=\int_{0}^{\infty} n^{h-1} e^{-n} d n$. The Schultz-Zimm distribution reduces to the exponential Flory distribution when $h=1$, which is another commonly used distribution for MWD. The 
Table 1: MWD model parameters

\begin{tabular}{l|l|l}
\hline Parameter & Unit & Value \\
\hline$K_{d}$ & $\mathrm{~min}^{-1}$ & $9.48 \times 10^{16} \exp (-30798.5 / r T)$ \\
$K_{i}$ & $L \cdot \mathrm{mol}^{-1} \cdot \mathrm{min}^{-1}$ & $0.6 K_{d}$ \\
$K_{p}$ & $L \cdot \mathrm{mol}^{-1} \cdot \mathrm{min}^{-1}$ & $6.306 \times 10^{8} \exp (-7067.8 / r T)$ \\
$K_{t r m}$ & $L \cdot \mathrm{mol}^{-1} \cdot \mathrm{min}^{-1}$ & $1.386 \times 10^{8} \exp (-12671.1 / r T)$ \\
$K_{t}$ & $L \cdot \mathrm{mol}^{-1} \cdot \mathrm{min}^{-1}$ & $3.765 \times 10^{10} \exp (-1680 / r T)$ \\
$V$ & $L$ & 3.927 \\
$F$ & $L \cdot \mathrm{min}^{-1}$ & 0.0286 \\
$T$ & $K$ & 353 \\
$I^{0}$ & $m o l \cdot L^{-1}$ & 0.0106 \\
$M^{0}$ & $m o l \cdot L^{-1}$ & 4.81 \\
$r$ & $\mathrm{cal} \cdot \mathrm{mol}^{-1} \cdot K^{-1}$ & 1.987 \\
$C$ & & {$[0.3,0.7]$} \\
\hline
\end{tabular}

two parameters of the Schultz-Zimm distribution function can be calculated by the leading moments of polymers as

$$
\begin{aligned}
h & =\frac{Z_{1}^{2}}{Z_{0} Z_{2}-Z_{1}^{2}} \\
M_{n} & =Z_{1} / Z_{0}
\end{aligned}
$$

In summary, the dynamic MWD modeling includes the following two steps:

S1: Get $Z_{0}, Z_{1}, Z_{2}$ from simultaneous integration of ODEs (42)-(49);

S2: Calculate $h$ and $M_{n}$ from (51) and (52), and formulate MWD by (50).

This first-principle model can be regarded as a soft sensor for MWD measurement, from which the input-output data pairs can be produced for B-spline PDF modeling. Model parameters are listed in Table 1. In this simulation, the chain length of the polymer is from 1 to 2000. The initial conditions for ODEs in (42)-(49) are set up as follows: $I(0)=0.0020$, $M(0)=2.2620, \psi_{0}(0)=0.0000, \psi_{1}(0)=0.0000, \psi_{2}(0)=0.0079, Z_{0}(0)=$ $0.0014, Z_{1}(0)=0.6240, Z_{2}(0)=425.8050$. These initial condition values are calculated from static model simulation at a given control ratio $C$.

A total number of $10 \mathrm{~B}$-spline basis functions are used in the B-spline approximation. Each B-spline function is a parabolic function represented 


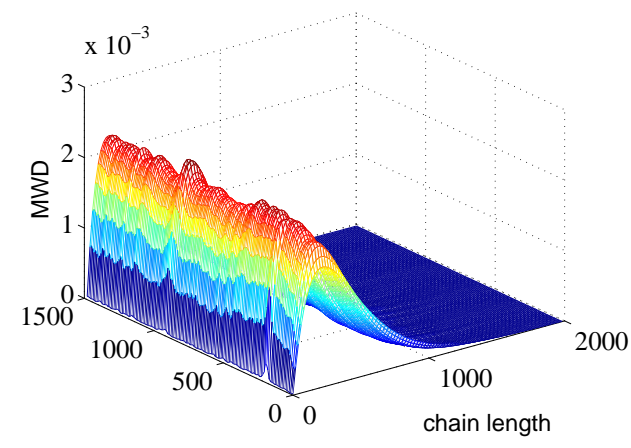

(a) Data for modeling

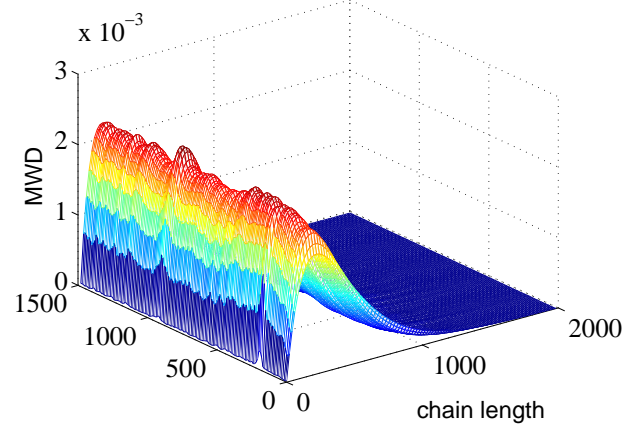

(b) Model prediction

Figure 2: MWDs from the first-principle model and the B-spline approximation model

as follows:

$B(i, j)= \begin{cases}-2 / w(i)^{2} \cdot(j-y(i)-w(i) / 2)^{2}+1 / 2, & y(i) \geq j \geq y(i)+w(i) \\ 0, & \text { otherwise }\end{cases}$

where $j=1,2, \cdots, 2000$ stands for the chain length of dead polymers in MWD, and

$$
\begin{aligned}
y(i) & =1+200(i-1), i=1,2, \cdots, 10 \\
w(i) & = \begin{cases}220, & i=1,2, \cdots, 9 \\
199, & i=10\end{cases}
\end{aligned}
$$

Here $i$ refers to the $i$ th B-spline basis function, $w$ is the parameter for Bspline width. The double-loop RLS algorithm in Section 2.3 is applied to estimate model parameters. Fig. 2(a) shows the 1500 sets of created MWD data used for establishing the RSR B-spline PDF model. Fig. 2(b) illustrates the B-spline modeling result regarding MWDs versus time and chain length. The MWD modeling error, defined by $\gamma_{1}(y, k)-\gamma_{2}(y, k)$ between two PDFs, is illustrated in Fig. 3. The scale of Fig. 3 is two-order lower than the MWD modeling data and this shows a good modeling quality.

\subsection{Shaping of $M W D$ by predictive output PDF control}

The initial MWD and the desired target MWD are set to be the same for the three control algorithms. The initial input level is set to be $C=0.5$ and the initial MWD is determined accordingly at $C=0.5$. The target 


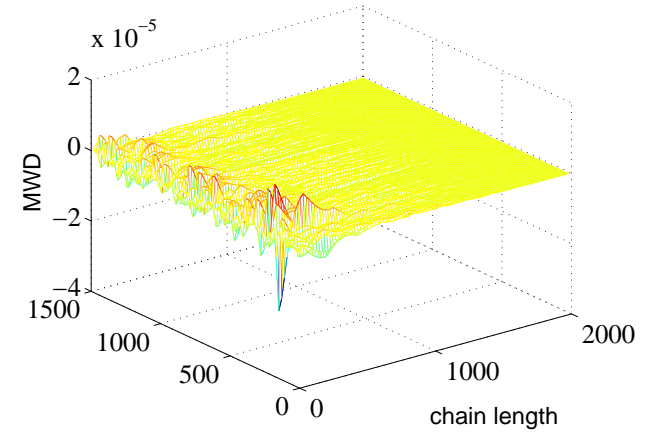

Figure 3: MWD modeling error

distribution is set corresponding to a control level of $C=0.6$. In all three control algorithms, the performance indexes for optimization consist of two terms, one for output PDF tracking performance and one for penalty of the control cost weighted by a $\mathrm{Q}$ factor. The penalty term needs to be tuned to achieve a balance between a good tracking performance and a low cost of control.

We first apply the standard output PDF control to this system, in which the weighting factor for control input is tuned to be $Q_{0}=0.0005$. We then implement the two predictive controllers by setting the model predictive horizon to be $p=9$, the control predictive horizon to be $l=5$, and $Q=0.0106 I_{5 \times 5}$ for both controllers. For the constrained MPC, the upper and lower bounds for control input are set to be $C \in[0.3,0.7]$. An SQP algorithm is adopted to solve the constrained nonlinear optimization problem and the fmincon() function is used in Matlab simulation. In the standard control and the nonconstrained predictive control, a hard-bound of $C \in[0.3,0.7]$ is applied to the control implementation considering the feasible operating conditions for this system, i.e., a 'saturated' $C$ is applied whenever the calculated control input is beyond this boundary domain. In order to compare the MWD tracking performance for each control algorithm, the following performance index

$$
J_{t}(k)=\int_{a}^{b}\left(\sqrt{\gamma(y, k)}-\sqrt{\gamma_{g}(y)}\right)^{2} d y
$$

is introduced to quantify the instantaneous distance between the output MWD and the desired MWD.

With proper tunings, all three controllers demonstrate a function to drive 


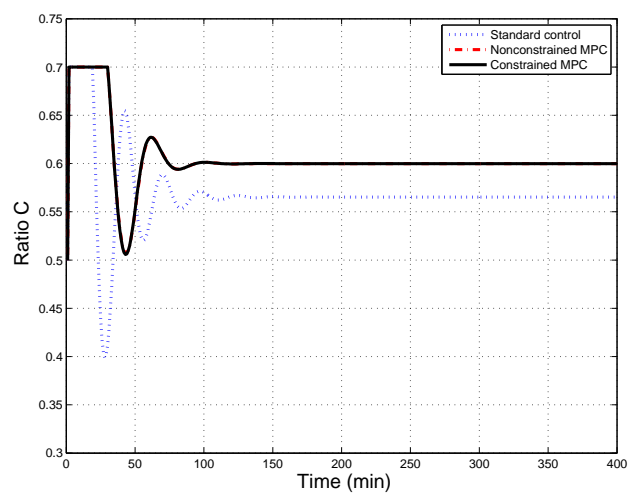

(a) Three control actions

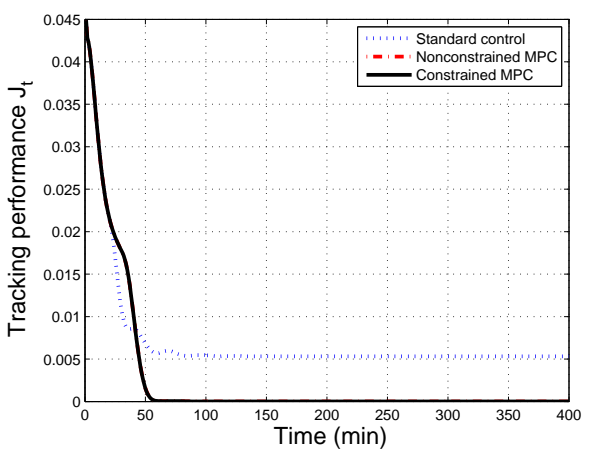

(b) Three tracking performances

Figure 4: Comparison of control input and tracking performance for 3 controllers $\left(Q_{0}=\right.$ $0.0005, Q=0.0106)$

the initial MWD to get closer towards the target MWD over the control process. The control input profiles for the three controllers are shown in Fig.4(a) and their tracking performances are illustrated in Fig. 4(b). The simulation results show that the non-constrained MPC has the same solution as the constrained MPC when a hard bound is imposed on the control input (in Fig.4(a) and Fig. 4(b), the curves corresponding to constrained and non-constrained MPCs are completely overlapped). This consistency is not surprising since in both cases the same level of constraints are considered, just handled in different manner. Fig. 5 illustrates MWDs at the initial state, the steady state and the desired target state under the standard PDF control and the predictive PDF controller, respectively. The dynamic evolution of MWDs under the predictive control (constrained and non-constrained) is demonstrated in Fig. 6 .

Under the standard output PDF control, it can be seen that there is a clear gap between the MWD at the steady state and the target MWD (Fig. 5(a)), while this steady-state MWD tracking error is much smaller (almost none) with predictive control. In Fig. 5(b), the final MWD curve overlaps with the target PDF curve. The steady-state performance can also be inspected from the control input profiles in Fig. 4(a), where for the two predictive control algorithms the control input signals almost reach the target level of $C=0.6$, when convergent, but the standard control input stays away from the target value at the steady state.

Taking the standard output PDF control, if we attempt to reduce the 


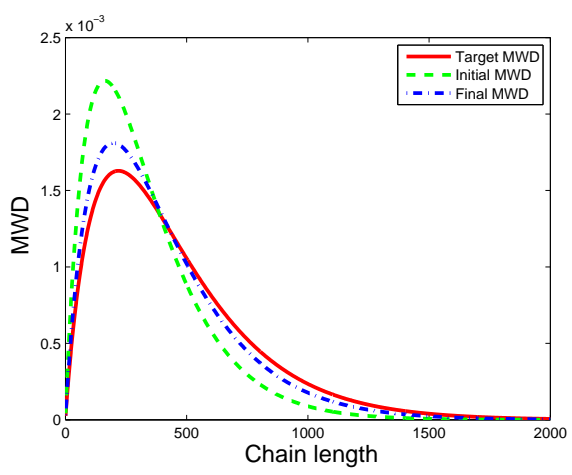

(a) Standard control

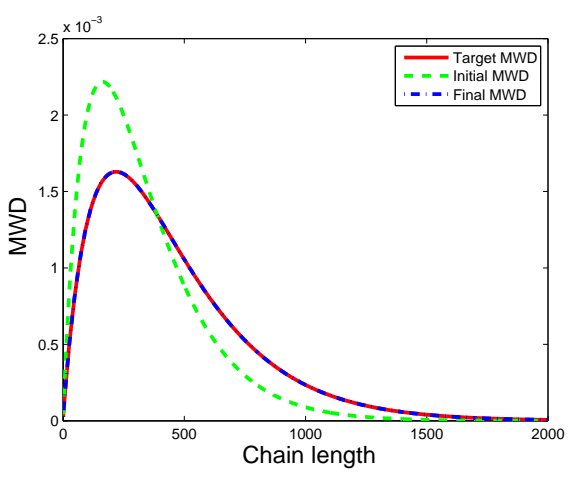

(b) Model predictive control

Figure 5: Initial, final and target MWDs under standard and predictive PDF control

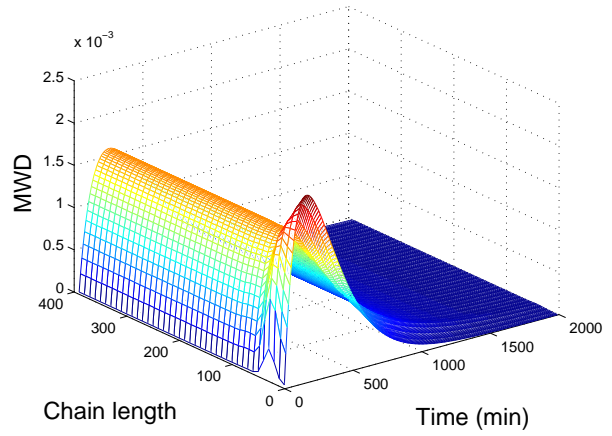

Figure 6: Dynamic evolution of MWDs under predictive control 


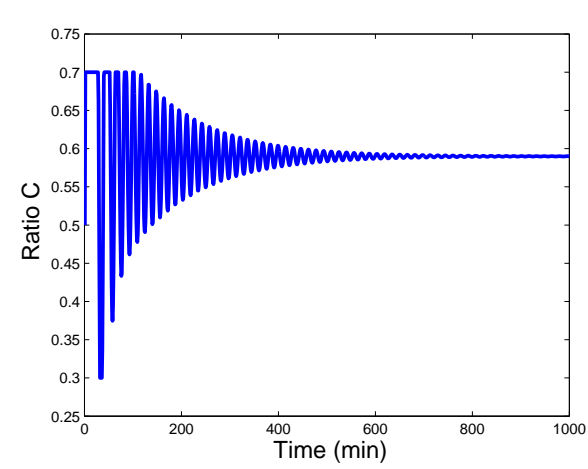

(a) Control action

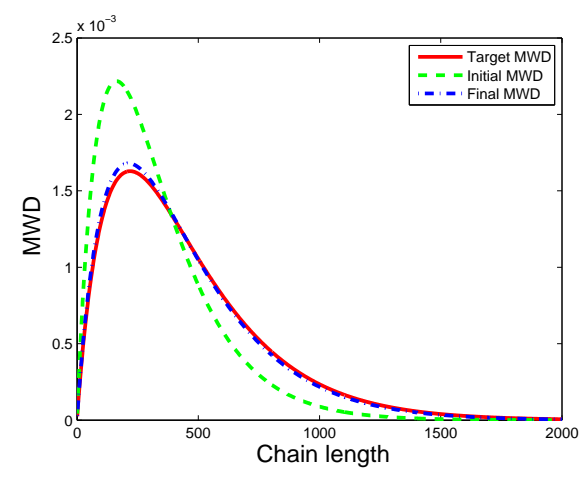

(b) Initial, final and target MWDs

Figure 7: Standard output PDF control with $Q_{0}=0.00014$

tracking error to the similar level that is achieved by predictive control, then more efforts need to be put on the tracking part of the performance function (35), which means paying a larger price in control to achieve improved tracking performance. To this end, $Q_{0}$ is reduced from 0.005 to 0.00014 , the simulation results of standard control is shown in Fig. 7. It can be seen that in this case, the MWD tracking error at steady state is reduced compared with the results in Fig. 5(a), but the control signal has serious oscillations and it takes a much longer time to reach the steady state.

As discussed in the design of all three output PDF controllers, the output PDF tracking performance is of the major concern, but the cost of control also needs to be considered for practical reasons. Compared with the standard output PDF control, the predictive control algorithms are more capable to achieve a balance between a good tracking performance and a reasonable control action. Also the constraints on control are included in the optimization process of the constrained predictive PDF control.

\section{Conclusion}

In this work, model predictive PDF control is investigated to shape MWD in a case study polymerization process through closed-loop feedback control. A generic modeling algorithm is proposed which can be used to establish a parameterized output PDF model using collected data from control input and output PDF. The RSR B-spline model is employed to approximate the output PDF, at each time instance, for the reason that it naturally satisfies 
constraints on positiveness and integration nature for a PDF, which outperforms three other commonly used B-spline models, i.e., linear, square root and rational B-spline models. With the RSR B-spline model, the actual weights instead of the pseudo weights are used in the data-based modeling of a linear weight dynamic system, which avoids complex estimation of the pseudo weights from output PDF and therefore largely simplifies the modeling procedure. The parameterized input-output model is further developed into a predictive PDF model that has the function of predicting the full output PDF over a future horizon.

Both non-constrained and constrained predictive control are developed to drive the output PDF towards its target shape. For the non-constrained MPC design, an analytical solution is obtained for a system with linear weight dynamic, which is computationally convenient but may overlook possible saturation in control actions. For constrained MPC design, a constrained nonlinear optimization problem needs to be solved, which is not a trivial task for a control problem with a full shape expected in its output distribution. The integrated modeling and predictive control scheme is applied to simulation study of an exemplar styrene polymerization process to implement closed-loop MWD control. Simulation results demonstrate the effectiveness of the proposed scheme and show the strength of predictive PDF control over standard output PDF control.

The B-spline based modeling provides a general methodology to describe output PDF control systems which can decouple time domain and space domain (or PDF definition domain) efficiently. The space-varying characteristic of the output PDF is approximated by a nonlinear B-spline neural network and the time-varying nature is depicted by a weight dynamic model. Modeling such a system using measurement data is thus a very challenging task since both the static B-spline approximation and the dynamic weights development need to be conducted in the same scheme. In this work, we attempt to explore data-based modeling techniques for output PDF control systems. The 'measurement data' used in simulation study are produced from a first-principle MWD model that we developed in a previous work. To our knowledge, no other work on data-based modeling of output PDF systems from industrial systems or simulation has been reported.

Currently a linear weight dynamic model is used which is an approximation to a nonlinear system. Development of nonlinear dynamic models for output PDF systems based on measurement data will be more difficult, but not impossible especially when the nonlinear dynamics can be parameterized. 
In addition to B-spline modeling, alternative solutions for output PDF modeling could be direct use of output PDF calculated from partial differential equations, estimation of output PDF by unscented Kalman filter, etc.

While MPC shed a light on tackling the output PDF control problem, problems like how to tune the MPC parameters to improve the control performance and how to solve the constrained nonlinear optimization effectively need to be further investigated. Since the predictive PDF controller is nonstructured and gradient-based, the analysis of closed-loop stability is difficult to perform. Future work on development of structured controllers for output PDF control is under investigation. The fundamental challenge of controlling a full distribution dynamically using a few control inputs remains an open problem for further exploration.

\section{Acknowledgments}

This work is partially supported by the Fundamental Research Funds for the Central Universities (2014MS25), NSFC (No. 61374052, No. 61473025), and the open-project grant funded by the State Key Laboratory of Integrated Automation for Process Industry at the Northeastern University, China.

[1] T. J. Crowley, K. Y. Choi, Experimental studies on optimal molecular weight distribution control in a batch-free radical polymerization process, Chem. Eng. Sci. 53 (1998) 2769-2790.

[2] T. Takamatsu, S. Shioya, Y. Okada, Molecular weight distribution control in a batch polymerization reactor, Ind. Eng. Chem. Res. 27 (1988) 93-99.

[3] M. F. Ellis, T. W. Taylor, K. F. Jensen, On-line molecular weight distribution estimation and control in batch polymerization, AIChE J. 40 (1994) 445-462.

[4] T. J. Crowley, K. Y. Choi, Discrete optimal control of molecular weight distribution in a batch free radical polymerizaiton process, Ind. Eng. Chem. Res. 36 (1997) 3676-3684.

[5] A. Echevarria, J. R. Leiza, J. de la Cal, J. M. Asua, Molecular weight distribution control in emulsion polymerization, AIChE J. 44 (1998) 1667-1679. 
[6] T. L. Clarke-Pingle, J. F. MacGregor, Optimization of molecular weight distribution using batch-to-batch adjustments, Ind. Eng. Chem. Res. 37 (1998) 3660-3669.

[7] M. Vicente, C. Sayer, J. R. Leiza, G. Arzamendi, E. L. Lima, J. C. Pinto, J. M. Asua, Dynamic optimization of non-linear emulsion copolymerization systems open-loop control of composition and molecular weight distribution, Chem. Eng. J. 85 (2002) 339-349.

[8] H. Yue, H. Wang, J. F. Zhang, Shaping of molecular weight distribution by iterative learning probability density function control strategies, Proc. IMechE Pt I: J. Syst. Contr. Eng. 222 (2008) 639-653.

[9] H. Y. Wu, L. L. Cao, J. Wang, Gray-box modelling and control of polymer molecular weight distribution using orthogonal polynomial neural networks, J. Proc. Control 22 (2012) 1624-1636.

[10] G. Penloglou, E. Kretza, C. Chatzidoukas, S. Parouti, C. Kiparissides, On the control of molecular weight distribution of polyhydroxybutyrate in azohydromonas lata cultures, Biochem. Eng. J. 62 (2012) $39-47$.

[11] S. H. Shen, L. L. Cao, J. Wang, Predictive control of molecular weight distribution in polymerization reaction based on moment of MWD, CIESC J. (in Chinese) 64 (2013) 4379-4384.

[12] C. Kiparissides, Challenges in particulate polymerization reactor modeling and optimization: A population balance perspective, J. Proc. Control 16 (2006) $205-224$.

[13] J. Dimitratos, G. Eliabe, C. Georgakis, Control of emulsion polymerization reactors, AIChE J. 40 (1994) 1993 - 2021.

[14] T. J. Crowley, E. S. Meadows, E. Kostoulas, F. J. D. III, Control of particle size distribution described by a population balance model of semi-batch emulsion polymerization, J. Proc. Contr. 10 (2000) 419-432.

[15] J. Flores-Cerrillo, J. F. MacGregor, Control of particle size distributions in emulsion semibatch polymerization using mid-course correction polices, Ind. Eng. Chem. Res. 41 (2002) 1805-1814. 
[16] C. D. Immanuel, F. J. D. III, Open-loop control of particle size distribution on semi-batch emulsion copolymerisation using a genetic algorithm, Chem. Eng. Sci. 57 (2002) 4415-4427.

[17] H. Wang, Bounded Dynamic Stochastic Systems: Modelling and Control, Springer-Verlag, London, 2000.

[18] R. A. Eek, O. H. Bosgra, Controllability of particulate processes in relation to the sensor characteristics, Powder Tech. 108 (2000) 137-146.

[19] H. J. C. Gommeren, D. A. Heitzmann, J. A. C. Moolenaar, B. Scarlett, Modelling and control of a jet mill plant, Powder Tech. 108 (2000) $147-154$.

[20] R. D. Braatz, Advanced control of crystallization processes, Annual Rev. Contr. 26 (2002) 87-99.

[21] E. Aamir, Z. Nagy, C. Rielly, Optimal seed recipe design for crystal size distribution control for batch cooling crystallisation processes, Chem. Eng. Sci. 65 (2010) $3602-3614$.

[22] Z. K. Nagy, R. D. Braatz, Advances and new directions in crystallization control, Annu. Rev. Chem. Biomol. Eng. 3 (2012) 55-75.

[23] J. S.-I. Kwon, M. Nayhouse, P. D. Christofides, G. Orkoulas, Modeling and control of shape distribution of protein crystal aggregates, Chem. Eng. Sci. 104 (2013) $484-497$.

[24] X. B. Sun, H. Yue, H. Wang, Modelling and control of the flame temperature distribution using probability density function shaping, Trans. Inst. Measurement and Control 28 (2006) 401-428.

[25] J. L. Zhou, H. Yue, J. F. Zhang, H. Wang, Iterative learning double closed-loop structure for modeling and controller design of output stochastic distribution control systems, IEEE Trans. Control Syst. Technol., In press (2014).

[26] A. E. Abharian, A. H. Fadaei, Power probability density function control and performance assessment of a nuclear research reactor, Annals of Nuclear Energy 64 (2014) 11-20. 
[27] J. Y. Zhu, W. H. Gui, C. H. Yang, H. L. Xu, X. L. Wang, Probability density function of bubble size based reagent dosage predictive control for copper roughing flotation, Contr. Eng. Practice 29 (2014) 1-12.

[28] G. A. Smook, Handbook for Pulp and Paper Technologists, Angus Wilde Publications, Vancouver, 1998.

[29] M. Karny, T. Kroupa, Axiomatization of fully probabilistic design, Information Sciences 186 (2012) 105-113.

[30] C. X. Zhu, W. Q. Zhu, , Y. F. Yang, Design of feedback control of a nonlinear stochastic system for targeting a pre-specified stationary probability distribution, Probabilistic Engineering Mechanics 30 (2012) 20-26.

[31] M. Annunziato, A. Borzi, A fokker planck control framework for multidimensional stochastic processes, J. Computational and Applied Mathematics 237 (2013) 487-507.

[32] L. Guo, H. Wang, Stochastic Distribution Control Systems Design: A Convex Optimization Approach, Springer-Verlag, 2010.

[33] H. Yue, J. F. Zhang, H. Wang, L. L. Cao, Shaping of molecular weight distribution using b-spline based predictive probability density function control, in: Proc. 2004 Am. Control Conf., Boston, USA, 2004, pp. $3587-3592$.

[34] H. Wang, H. Baki, P. Kabore, Control of bounded dynamic dynamic stochastic distributions using square root models: an applicability study in papermaking system, Trans. Inst. Meas. Control 23 (2001) 51-68.

[35] H. Wang, H. Yue, A rational spline model approximation and control of output probability density functions for dynamic stochastic systems, Trans. Inst. Meas. Contr. 25 (2003) 93-105.

[36] J. L. Zhou, H. Yue, H. Wang, Shaping of output pdf based on the rational square-root b-spline model, Acta Automatica Sinica 31 (2005) 343-351.

[37] S. J. Qin, T. A. Badgwell, A survey of industrial model predictive control technology, Control Eng. Pract. 11 (2003) 733 - 764. 
[38] R. Findeisen, F. Allgower, An introduction to nonlinear model predictive control, in: 21st Benelux Meeting on Systems and Control, Veldhoven, Netherlands, 2002, pp. 1-23.

[39] E. F. Camacho, C. Bordons, Nonlinear model predictive control: An introductory review, in: R. Findeisen, F. Allgower, L. Biegler (Eds.), Assessment and Future Directions of Nonlinear Model Predictive Control, Springer-Verlag, 2007, pp. 1-16.

[40] B. Alhamad, J. A. Romagnoli, V. G. Gomes, On-line multi-variable predictive control of molar mass and particle size distributions in freeradical emulsion copolymerization, Chem. Eng. Sci. 60 (2005) 6596 6606.

[41] T. Kreft, W. F. Reed, Predictive control of average composition and molecular weight distributions in semibatch free radical copolymerization reactions, Macromolecules 42 (2009) 5558-5565.

[42] J. F. Zhang, H. Yue, J. L. Zhou, A new pdf modelling algorithm and predictive controller design, in: Prepr. 10th IFAC Symp. Dynamics. Contr. Proc. Syst. (DYCOPS2013), Mumbai, India, 2013, pp. 271-276.

[43] H. X. Wu, J. Hu, Y. Xie, Characteristic model-based all-coefficient adaptive control method and its applications, IEEE Trans. Syst., Man, Cybern., pt C: Appl. Rev. 37 (2007) 213-221.

[44] D. Q. Shu, Predictive Control and Applications, Mechanical Industry Publishing Company, Beijing, 1996.

[45] H. J. Angerman, The Phase Behavior of Polydisperse Multiblock Copolymer Melts: a Theoretical Study, PhD thesis, University Library Groningen, 1998. 